\title{
Chatbot Developments in The Business World
}

\author{
Azani Cempaka Sari*, Natashia Virnilia, Jasmine Tanti Susanto, Kent Anderson Phiedono, Thea Kevin Hartono \\ Computer Science Department, School of Computer Science, Bina Nusantara University Kav 21, Alam Sutera Jalan Jalur Sutera Barat \\ Panunggangan Timur, RT.001/RW.004, Panunggangan, Kec. Pinang, Kota Tangerang, Banten, 15143, Indonesia
}

\begin{tabular}{l} 
A R T I C L E I N F O \\
\hline Article history: \\
Received: 23 June, 2020 \\
Accepted: 12 October, 2020 \\
Online: 24 November, 2020
\end{tabular}

Keywords:

Chatbot

Artificial Intelligence

Natural Language Processing

(NLP)

Business

AIML

\begin{abstract}
A B S T R A C T
Chatbot is an application that is designed to help humans communicate with machines. NLP and API.AI are some of the components that are crucial in making chatbot as they help chatbot in performing chatting operations. Chatbot could be implemented in many aspects of life such as education, social media platforms, games, even in business. Business has always been an important part of human life and it is irreplaceable. As the era evolves, the business industry is also getting modernized and automated which resulted in implementing the latest technology into the industry like chatbot. In the context of the business industry, chatbot can be used as an assistant that will help dealing with the customer service section as well as giving the latest information about the business. This study aims to determine how the chatbot gives impact to the business as well as the owners, what kind of features that the chatbot needs, and how the chatbot should be developed so that it will be compatible in the business environment. The research was conducted using two methods which are literature review and survey: questionnaire. The result of the research conducted are the impacts that the owner gets by using chatbot in their business, a set of features that are relevant in developing chatbot and recommendation for further implementation.
\end{abstract}

\section{Introduction}

Chatbot is an application or service that can interact with users by communicating using text or audio. The first chatbot produced was ELIZA [1] in which this chatbot could communicate using simple responses with the user. The usage of chatbot nowadays is inclining significantly that it is easy to find chatbot in web sites with each different functionality. Chatbot both on applications and web sites usually give services like giving important information, helping users in operating data, and supporting business processes. Surprisingly, chatbots can fit in every kind of website or program, even gaming sites. Thus, it is easy to say that chatbot is also gaining popularity in the business environment since chatbot could confirm that messages or complaints from customers can be handled fast and accurately. In addition, companies that use chatbot could cut down on their operational fees [1] and also their business could be active for 24 hours every day. In technology development nowadays, there are lots of research that uses chatbot as virtual assistant or shop assistant. Chatbots are programmed so that they can interact with users using natural human language. These days, there are many

\footnotetext{
*Corresponding Author: Azani Cempaka Sari, kent.phiedono@binus.ac.id
}

chatbots that are based on AIML or Artificial Intelligence Markup Language like virtual assistants such as Voice Actions, Skyvi, Iris, and Call Mom (Pandorabots.com). AIML is often used for programming the artificial intelligent software of chatbot or also known as knowledge base [2, 3]. Technology these days is developing so fast that it is now reaching to all kinds of people and covering different aspects in life like health, business, education, and many more. This is because information plays an important role in this globalization era. Everything including daily activities needs information before doing it and every action that was taken is demanded to produce another information that is helpful for many people. Thus, as technology is something that makes information reachable, technology including chatbots are developing and expanding fast and the demand of technology is for sure high. In Indonesia, chatbots are becoming popular as they can communicate to customers as if they are literally talking naturally and they can answer different questions faster than humans. It is usually implemented to support the company's service as chatbots will be likely to increase the service rate. Moreover, chatbot will not forget any information unlike humans who will be most likely to forget the information that is needed to be delivered. Thus, in this research we are planning to know 
whether chatbots are actually important in running businesses, if it is needed in the business, and what kind of chatbot model that is suitable for supporting a business.

\section{Literature Review}

The basic mechanism of chatbot starts with messages sent by the user. The message is then processed by NLP (Natural Language Processing), and chatbot responds by replying to the message according to the existing database [4].

\subsection{Chatbot}

Chatbot is a character that communicates with its user or people using natural human language in chatting forums such as messenger, instant web, email, usenet, forum, web, and even using speech like telephone. Chatbot has so many aliases and some of them are chat robot, bot, chatter bot, bot chatting, chatterbox, V-Host, V-People, agent, and virtual human. Chatbot can also be connected to an avatar or animation that includes speech synthesis so that chatbot could be seen as something livelier as we, human beings, could see what it is and what it is speaking. To improve the service given, chatbots are programmed so that it could analyze words, phrases, and the construction of sentences of inputs so then it could predict the users' personality, preferences of products, and give responses according to what it had analyzed. While doing the conversations, chatbots usually are collecting previous responses and inputs so their future responses could vary and it will also improve the quality of its future responses. Giving personal recommendations is an example of the result in collecting data from previous conversations.

In the context of e-commerce, chatbots can handle conversations with customers and help them in the process of selling which are expected to give positive effects toward online businesses [5]. It is believed that experienced users feel more satisfied when communicating with virtual communicating agents rather than communicating with inexperienced users in all kinds of aspects that were evaluated including impression, control, effectiveness, navigation, learning, and many more [5].

There are 3 things that form a combination and this combination is the main pillar of chatbot. The 3 things are:

\subsubsection{User Interface}

User interface referencing to the display interface of a chatbot that bridges between user and chatbot to interact with each other. User interface has to give the best experience to the users when interacting with the user. Without it, chatbot will be useless as it is not convenient to use. The interface includes the text's font, background color or theme, animation, images, and navigation tools.

\subsubsection{Artificial Intelligence}

AI or Artificial Intelligence is like the core of the whole chatbot application because it is the main brain where everything is processed [6]. The chatbot application could understand inputs when interacting with users because of this. Chatbot is the expansion of the artificial intelligence field so that is why artificial intelligence is very crucial for the whole development of chatbot.

\subsubsection{Integration}

Integration with other applications or systems can enrich the chatbots' features. Chatbot could get additional or supporting materials and information by integrating it with another system. Thus, integration could improve the user experience and satisfaction. Although it is not as important as artificial intelligence, integration plays a major role too in promoting the usefulness of chatbot.

In a business environment, chatbot can help solve problems regarding communicating with users or customers especially in terms of service and experience when interacting. Thus, chatbot is very effective particularly in problems that are fixated, specific, and predictable. In the rapid business development environment, chatbot becomes an alternative solution that can help customers to reach and interact with business.

\subsection{Platform API.AI}

API.AI is a platform that provides NLP (Natural Language Processing) and NLU (Natural Language Understanding) services. These services are used to make chatbot smarter and can understand the purpose of what is asked by the user. Natural language processing is one of the disciplines of Artificial Intelligence that focuses on human and computer interactions through natural language that humans use. In NLP the goal to be achieved is the ability of an NLP system to have natural language knowledge both from the sentence structure, the meaning of the word and the purpose of a sentence. While NLU itself is a subfield of NLP, where the focus of the purpose of NLU itself is to make an understanding of a sentence and conduct semantic analysis. API.AI itself does not support the Indonesian knowledge domain but has a lot of knowledge domains in English. Domain is a collection of knowledge and data structures. Within API.AI itself many knowledge bases have been embedded in this API.AI service system. The collection of knowledge contained in this service includes musician entities, airports, etc. However, the absence of Indonesian language support in this service does not mean this service cannot be used by researchers who speak Indonesian language [7].

\subsection{Chatbot Type (Based on its functionality}

\subsubsection{Business Management Chatbot}

This chatbot type is usually found in business environments. Aside from communicating with users, there are some other abilities which are processing business process data and giving information related to certain business. Usually the chatbot is implemented in applications or websites that are related to business and it acts as a supporting application. Chatbots that can be classified as business management chatbot are CardBot, Naver TalkTalk, SuperAgent, and many more $[8,9,10]$.

\subsubsection{Game Chatbot}

This kind of chatbot is the most popular chatbot type among youngsters, especially gamers. It is usually used for entertainment as it is used when users are playing games. The chatbot is usually used when playing multiplayer games but it is compatible with single player games too. One of the examples is SimSimi in which we can use it in mobile and web platforms. 


\subsubsection{Music Chatbot}

Just like its name, this chatbot type is used for playing music. The chatbot is actually pretty easy to use as the chatbot will ask the user to input their choice of songs and the chatbot will automatically search the song in platform YouTube or any accessible platform then it will play the song. This chatbot is usually found in chatting applications that have voice related features in it like Discord application. The chatbot examples are Groovy, FredBoat, and Rythm.

\subsubsection{Assistant Chatbot}

Assistant Chatbot just like its name means that it helps users in doing daily activities just like how secretaries in real life do [11]. Telling weather forecasts, planning plans, setting important alarms, and asking to call someone are some of the features that this type of chatbot has. The examples of this kind of chatbot are Google Assistant, Siri from Apple, Alexa from Amazon, and Cortana from Microsoft [11].

\subsubsection{Education Chatbot}

Education chatbot is a chatbot that is used to help users in learning. This type of chatbot is usually implemented in the education field. The benefits of using this chatbot is that it can cut down operational fee and save time when learning. The examples of this kind of chatbot are Duolingo, Gengobot [4].

\subsection{Chatbot Examples}

\subsubsection{Telegram Bot}

Telegram Bot is a social media bot that many people installed. This bot gives user feature API (Application Programming Interface) this feature which allow chatbot exist. This feature used for direct Chatting with using chatbot that made user can make chatbot suitable as user wish. Telegram bot started from registration about bot used for chatbot [7].

\subsubsection{Pandorabots}

Pandorabots is an online service which allows developers to host and deploy Chatbot. This chatbot has 225.000 Developers registered and tools already help 300.000 chatbot. Pandorabots using AIML(Artificial Intelligence Markup Language) which is a standard for writing code in chatbot. This chatbot offers many tools including 'Artificial Intelligence as a Service' these tools provide access API to platform bot hosting and pandorabots playground : a development environment used for made chatbot. Pandorabots are working on constant and have very large active communication. Pandorabots obey the standard for making chatbot. Up until now, open source software has been used for building pandorabots. Pandorabots are also known to be using AIML as knowledge content markup language [12].

\subsubsection{CardBot}

CardBot is a chatbot which is a result of a research by MengChieh Ko and Zih- Hong Lin in his research entitled CardBot: A Chatbot for Business Card Management with the additional function of being able to read data from business cards with OCR techniques collaborated with cameras and also has a function to organize the data it has acquired into a knowledge base [10]. The data that has been obtained will be managed, combined and stored so that when a user requests data from that already obtained chatbot can immediately retrieve the data to the user. This chatbot uses NLP development technique in its development [10] and includes a type of chatbot for business management.

\subsubsection{SuperAgent Chatbot}

SuperAgent Chabot is a chatbot with features that can read data from web pages (about products) and can answer questions from users based on data available on these web pages [9]. In addition to these features, chatbot can carry out regular conversations (chit-chat) with users and the knowledge base of this chatbot can be added by the developer as well. This chatbot was made with machine learning techniques, NLP, and using the DSSM model for attribute matching and the first candidate will be the result of the response of this chatbot [9]. This chatbot is used as an extension on the web and can be used on any website (not just linked to a web or application) [9].

\subsection{Natural Language Processing (NLP)}

This subfield of artificial intelligence is a technique that helps computers understand human language. Using NLP, machines can make sense of unstructured online data so that we can gain valuable insights. NLP primarily comprises natural language understanding (human to machine) and natural language generation (machine to human). This article will mainly deal with natural language understanding (NLU). In recent years there has been a surge in unstructured data in the form of text, videos, audio and photos. NLU aids in extracting valuable information from text such as social media data, customer surveys, and complaints.

\subsubsection{Common Techniques used for extracting information}

\section{Named Entity Recognition}

This technique is one of NLP techniques that is used to extract texts, name and categorize them into a certain topic or context. Named entity recognition or NER is serving as important targets for most language processing systems because they usually carry key information in a sentence [13]. Accurate named entity recognition can be used as a useful information source for different NLP applications. There are lots of topics that NER can identify but there are 3 classes that NER systems use the most: person (PER), location (LOC), and loosely defined miscellaneous (MIS) [13].

\section{Sentiment Analysis}

Sentiment analysis or also known as opinion mining is a technique where it aims to determine the attitude of a writer with respect to some topic or the overall contextual polarity of a document [14]. This technique differentiates texts or documents into two main parts: objective (facts) and subjective (opinions). Here are text examples on both objective and subjective:

Objective: I bought a book.

Subjective: The paper was nice.

Sentiment could be identified through three methods which are manual opinion words generation, dictionary-based opinion 
words generation and corpus-based opinion words generation. Manual opinion words generation is a method in which we collect the opinion words manually and it is less effective than the other two. The dictionary-based opinion words generation is a method in which we use a seed list and grow the list using words from the dictionary until there are no new opinion words. The last method is a method in which it depends on syntactic or co-occurrence patterns in large text corpora.

In sentiment analysis, opinion words are counted and grouped into 3 categories: positive, negative, and neutral. Opinion words such as "good", "amazing", "great" are considered positive meanwhile "bad", "suck", "terrible" are considered negative. In sentences, every word that is considered positive will be graded as $(+1)$ while negative opinion words will be graded as (-1). If the end result is 0 then the text will be considered neutral, if the result is above 0 then the text will be considered as positive text, and if the result is below 0 then the text will be considered as negative text [14].

\section{Text Summarization}

Just like the topic's name, this technique aims to create a summary of a certain document that contains the most important information of the original one [15]. The technique mainly involves large chunks of text such as news articles.

There are two approaches to text summarization: abstraction and extraction. The extraction method works by identifying important sections of the text and generating them verbatim; thus, they depend only on extraction of sentences from the original text [16]. Meanwhile, the abstraction method aims to produce important material in a new way. Since existing abstractive summarizers often rely on an extractive preprocessing component to produce the abstract of the text [16], the paper will be focused on discussing the extractive summarization method. There are three main tasks of extractive text summarization which are to construct an intermediate representation, give sentence score, and summary sentence selection.

\section{Aspect Mining}

Aspect mining is a technique that is similar to sentiment analysis but focuses mainly on identifying different aspects in text. There are two major tasks in aspect mining which are aspect extraction and aspect sentiment classification. Process of identifying the opinion words from the given sentence is called aspect extraction and categorizing the extracted opinion words into one of the polarity scales is called aspect sentiment classification [17]. The polarity scales in aspect mining are broad but we can narrow them into 3 major scales: positive, negative, and neutral.

\section{Topic Modeling}

Topic modeling is a technique where it aims to give the reader better understanding by identifying natural topics in the text. There are four most popular methods in topic modeling which are Latent Semantic Analysis (LSA), Non-Negative Matrix Factorization (NNMF), Probabilistic Latent Semantic Analysis (PLSA), and Latent Dirichlet Allocation (LDA) [18].
LSA is an algebraic method that presents the semantic space of documents. The more semantic the relationship, the contextual usage will come closer. LSA is based on single value decomposition (SVD). NNMF on the other hand is another reduction technique where there are negative numbers in the dataset which is a problem and are addressed by placing nonnegativity constraints on the data model. PLSA is another technique of reduction for detecting semantic co-occurrence of terms in text mining based on bag of words (BOW). PLSA uses a probabilistic framework in a corpus. Finally, LDA is an approach to capture significant inter as well as intra document statistical structure by mixing distributional assumptions that document could arise from multiple topics. The topic is defined as distribution over a vocabulary. This approach is based on the Definneti theorem.

\subsubsection{Advantages of NLP}

Natural Language Processing can perform a large-scale analysis which can help machines perform language-based tasks such as reading text, identifying topics or what is important, or even extracting sentiment in a text. Moreover, NLP can perform these tasks in such a short time while still applying consistent and unbiased criteria. Thus, it can help people in doing these kinds of tasks faster and more accurately.

Other than large-scale analysis, Natural Language Processing has increased the level of sophistication in engineering because generic engines now can deliver semantic representations for sentences or sentences for representations [19]. Natural Language Processing also makes engines to be more user-friendly since it is highly expressive, highly flexible, and highly representative of reality [20]. On the other hand, NLP helps to bridge the gap between machines and humans because it can decipher information to computers and interpret the way people talk.

\section{Research Method}

\subsection{Literature Review}

Literature review is a process in which researchers conduct surveys from scientific sources on a specific topic. In this study, the collection of scientific sources based on international journals, international conferences, and the results of research on chatbots in the business world. All scientific sources are obtained through the internet namely, Google Scholar, ScienceDirect, Academia.edu, and the IEEE website. The sources that had been collected will be analyzed and summarized into important points.

\subsection{Survey: Questionnaire}

The survey is classified as qualitative data collection. A qualitative approach with case studies is applied in this study where qualitative view of field conditions by collecting data from informants. The survey was done online using Google Forms and each of the results were checked and analyzed thoroughly. After the checking, the results were then summarized as points.

The questions mainly ask about people's perspective on chatbot, its effectiveness, and its features to be added on the chatbot in a business-related environment. This survey method is reliable as the questions are based on this high technology era situation and respondents from all over the world might have the 
same responses towards the same question. Thus, this method may produce results that are consistent and reproducible. For the validity of the method, the results would correspond well to the questions and would produce accurate results as the questions were made and distributed to people who are exposed to technology and business at the same era of time.

\section{Results}

\subsection{Questionnaire and Literature Review Result}

Based on that questionnaire results, we get that most of the respondents feel that chatbot will be helpful for online shop owners or sellers when handling customers (91 out of 100 respondents). Respondents also feel that an up-to-date answer from the seller is important thus, we can conclude that chatbot will be useful since it responds faster than humans (98 out of 100 respondents). Although most of the respondents feel that chatbot may be able to handle an online shop alone (39 out of 100 respondents) or with human's help (38 out of 100), there are still some respondents who feel that it is better for an online shop to be handled by the seller himself ( 27 out of 100 ). From the results shown above, we can conclude that the chatbot which is suitable in the business environment is a fast-responding chatbot with upto-date data regarding the products or services. Although the idea of having a chatbot handling the online business alone is wonderful, we still believe that the seller plays a major role in the online business and it is better to have both chatbot and the seller handling the online business. In conclusion, chatbots could be accepted by people in business and should be helping the seller instead of handling the business alone. These questionnaire results are from our online questionnaire that we shared on online messaging platforms like LINE application and WhatsApp. The respondents vary from university students (Binus University Alam Sutera) to online shop owners or sellers in Indonesia.

On the other hand, there are some suitable features of a chatbot for business environments based on our literature review. We found out that the SuperAgent chatbot researched by Lecu, Shaohanh, Fuwei, V-Chutan, V-Chadu, and Mingzhou can identify texts whether it was about fact Q\&A (information detail about products or services), text Q\&A (opinion about the products or services), or chatting [9]. From another journal titled Chatbot Using A Knowledge in Database which was researched by Bayu Setiaji and Ferry Wahyu Wibowo has great features for chatbot especially for online business that need to be pre-trained by a knowledge base. Moreover, it can be used in any language but with some boundaries so chatbots can be more flexible [21]. Lastly, Intellibot which was researched by Mohammad Nuruzzaman and Omar Khadeer Hussain can set a great example for chatbots because it uses a movie as its knowledge base for answering chit-chats and also another personalized knowledge base for questions about products or services [22]. There are some other chatbots that we considered as suitable for the business environment with each feature that will be described in Table 1: Chatbot Description Table.

Table 1: Chatbot Description Table

\begin{tabular}{|l|l|}
\hline Chatbot & Description \\
\hline Chatbot [21] & $\begin{array}{l}\text { Chatbot created using database as its knowledge } \\
\text { base (RDBMS MySQL), pre-trained chatbot, }\end{array}$ \\
\hline
\end{tabular}

\begin{tabular}{|c|c|}
\hline & $\begin{array}{l}\text { using bigram method for processing text and } \\
\text { identifying response, can be used in any language } \\
\text { but with some boundaries, can understand similar } \\
\text { words, typo, or even different words and similar } \\
\text { meaning (responses are based on the data inside } \\
\text { the database). Refer to Fig. } 1 \text { for the example of } \\
\text { its usage. The text is in Indonesian but it mainly } \\
\text { talks about daily conversation. }\end{array}$ \\
\hline $\begin{array}{l}\text { Naver TalkTalk } \\
{[8]}\end{array}$ & $\begin{array}{l}\text { Naver TalkTalk chatbot is a chatbot with } \\
\text { cardbot-based interface and using RPA (Robotic } \\
\text { Process Automation) as its automation. The bot } \\
\text { is pre-trained because the bot will proactively ask } \\
\text { questions that is based on its data or knowledge } \\
\text { base that previously must be trained or if not the } \\
\text { chatbot will not be able to ask related questions. } \\
\text { The interface is using cardbot interface and it is } \\
\text { simpler to use and to understand what the bot is } \\
\text { trying to say. This chatbot is much more } \\
\text { compatible with business environment but sadly } \\
\text { no chit-chatting can be held in this type of } \\
\text { chatbot. Refer to Fig. } 2 \text { for its example of chatbot } \\
\text { usage and its cardbot-based interface. }\end{array}$ \\
\hline SuperAgent [9] & $\begin{array}{l}\text { SuperAgent chatbot is a chatbot that implements } \\
\text { machine learning and NLP (Natural Language } \\
\text { Processing). The chatbot is connected to business } \\
\text { environment and act as a marketing agent. The } \\
\text { bot is not pre-trained because it takes what was } \\
\text { written on the web page as its knowledge base } \\
\text { and will change when the web page is changed } \\
\text { too. It is flexible and easy to use as it is used as } \\
\text { an extension of an e-commerce web sites. } \\
\text { Although it is pretty good for business chatbot, } \\
\text { datasets from external can not be implemented as } \\
\text { it is not pre-trained. Refer to Fig. } 3 \text { to } \\
\text { differentiate between fact QA, text QA, and chit- } \\
\text { chat in SuperAgent chatbot. }\end{array}$ \\
\hline Intellibot [22] & $\begin{array}{l}\text { Intellibot for insurance needs, it is a pre-trained } \\
\text { chatbot that uses } 2 \text { datasets (Cornell movie and } \\
\text { insurance custom dataset) and it is dialogue- } \\
\text { based with NLP techniques implementation. The } \\
\text { result of this paper's research turns out that the } \\
\text { chatbot is more superior than the other bots } \\
\text { tested. Refer to Fig. } 4 \text { as the questions given by } \\
\text { the user and answers from the chatbots with its } \\
\text { strategy selection level ranges from } 1-5 \text { as } \\
\text { numbers of accuracy and relevancy with } 5 \text { as the } \\
\text { highest level. }\end{array}$ \\
\hline $\begin{array}{ll}\text { Chatbot } & \text { with } \\
\text { Business } & \\
\text { Intelligence } & \text { with } \\
\text { Big } & \text { Data } \\
\text { Integration } & \\
\text { (Enquiry } & \text { Bot) } \\
\text { [23] } & \end{array}$ & $\begin{array}{l}\text { The chatbot uses big data analytics for the } \\
\text { process of collecting, organizing, and analyzing } \\
\text { large data sets to discover hidden patterns or } \\
\text { unknown information. The chatbot uses AIML } \\
\text { for its knowledge base and it is a pre-trained } \\
\text { chatbot as its knowledge base is from the large } \\
\text { data set mentioned earlier. Refer to Fig. } 5 \text { on how } \\
\text { Enquiry Bot answers questions given however } \\
\text { the answers given are still not human-like. }\end{array}$ \\
\hline CardBot [10] & $\begin{array}{l}\text { CardBot is a chatbot that implements OCR } \\
\text { techniques with a goal to help people manage } \\
\text { business cards cleverly. It uses NLP for } \\
\text { processing the text and OCR to process the text } \\
\text { from physical to digital. The knowledge base is } \\
\text { based on what was taken by OCR and NLP thus, } \\
\text { it is not a pre-trained chatbot. Refer to Fig. } 6 \text { on } \\
\text { how CardBot read information with OCR } \\
\text { Technique and how the user can manage business } \\
\text { cards efficiently. }\end{array}$ \\
\hline
\end{tabular}




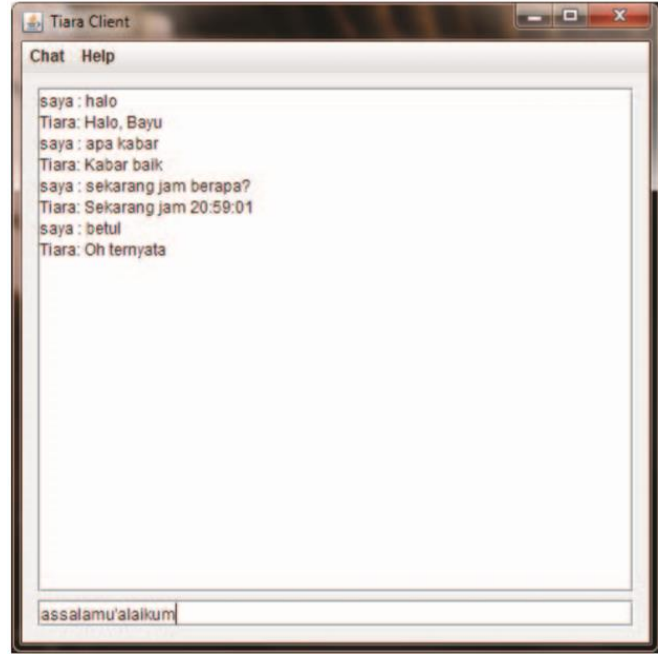

Figure 1: Chatbot
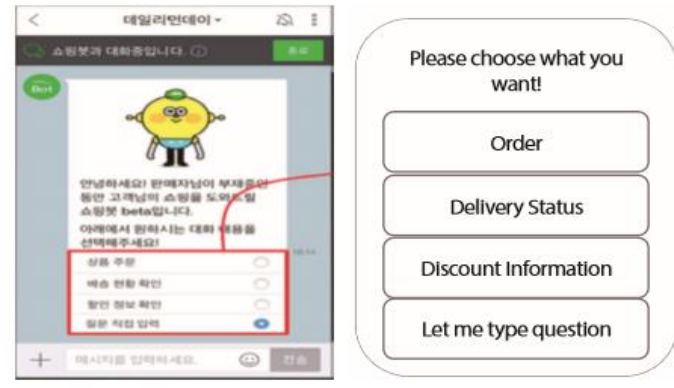

Figure 2. A Typical Cardbot Interface.

Figure 2: Naver TalkTalk Chatbot

\subsection{Natural Language Processing (NLP) Techniques Corresponding to The Result}

From the result of both questionnaire and literature review, we can conclude that the ideal chatbot for a business environment must be fast and accurate when answering questions with up-todate information. From what we had researched, NLP is commonly used in chatbots for text processing. Moreover, chatbots with NLP are fast and accurate when answering questions and their answers are mostly in context so NLP is the most compatible text processing method for developing business related chatbots [13]. There are a lot of techniques in NLP but Named Entity Recognition and Sentiment Analysis will be the most frequently used techniques in chatbots.

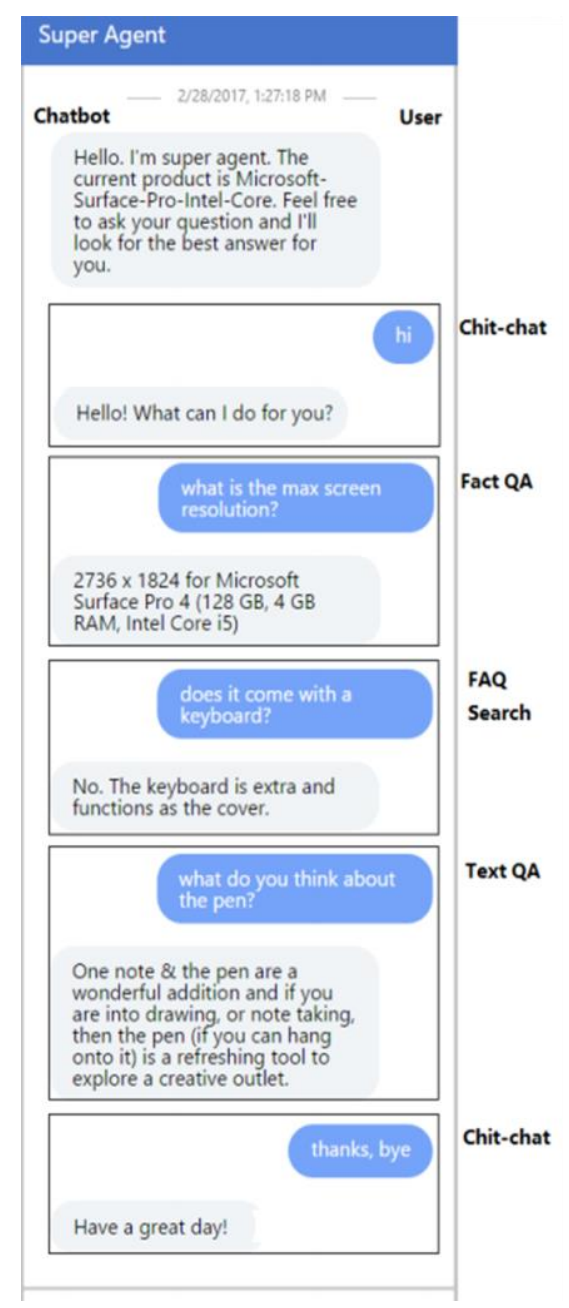

Figure 3: SuperAgent Chatbot

\begin{tabular}{|c|c|c|c|c|c|c|}
\hline \multicolumn{7}{|c|}{ Greetings } \\
\hline & User Input & RootyAl & ChatterBot & DeepQA & IntelliBot & $\begin{array}{l}\text { Strategy } \\
\text { selection }\end{array}$ \\
\hline & Bot Initialization: & $\begin{array}{l}\text { Hello, I'm Al } \\
\text { assistance. How } \\
\text { may I help you? }\end{array}$ & $\begin{array}{l}\text { Hi there, How } \\
\text { may I assist you? }\end{array}$ & $\begin{array}{l}\text { Hello, I'm Papaya. } \\
\text { I'm your assistant. }\end{array}$ & $\begin{array}{l}\text { Hello, I'm IntelliBot. Your } \\
24 / 7 \text { h assistance. How } \\
\text { may I assist you? }\end{array}$ & 1 \\
\hline 1 & Hello & Hello & Hello & Hello & Hello & 1 \\
\hline 2 & Good morning. & Good morning. & Good morning. & Good morning. & Good morning. & 1 \\
\hline 3 & My name is Nur. & $\begin{array}{l}\text { Nice to meet you, } \\
\text { Nur }\end{array}$ & Ok, Nur & $\begin{array}{l}\text { Ok. How can I } \\
\text { help you, Nur? }\end{array}$ & $\begin{array}{l}\text { Nice to meet you, Nur. } \\
\text { How may I help you? }\end{array}$ & 1 \\
\hline 4 & What is your name? & I don't know. & I don't have it. & I'm Papayay. & $\begin{array}{l}\text { My name is IntelliBot. } \\
\text { What yours? }\end{array}$ & 1 \\
\hline
\end{tabular}

Figure 4: Intellibot 


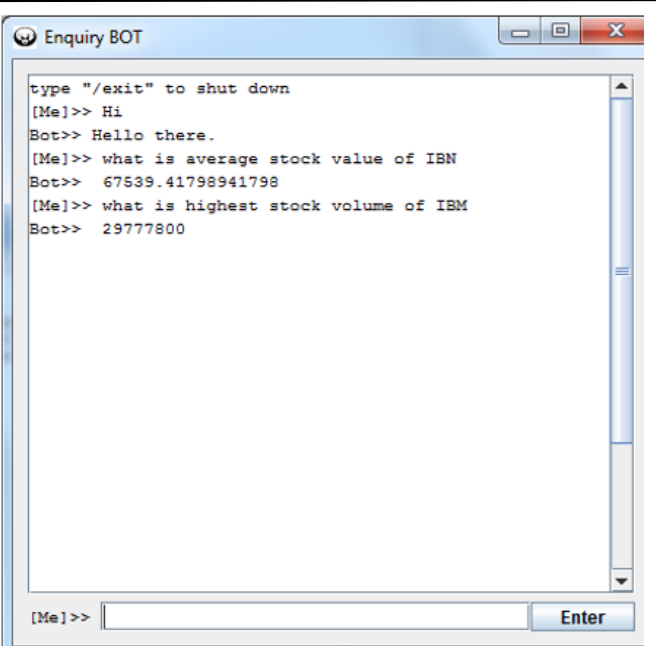

Figure 5: Enquirybot

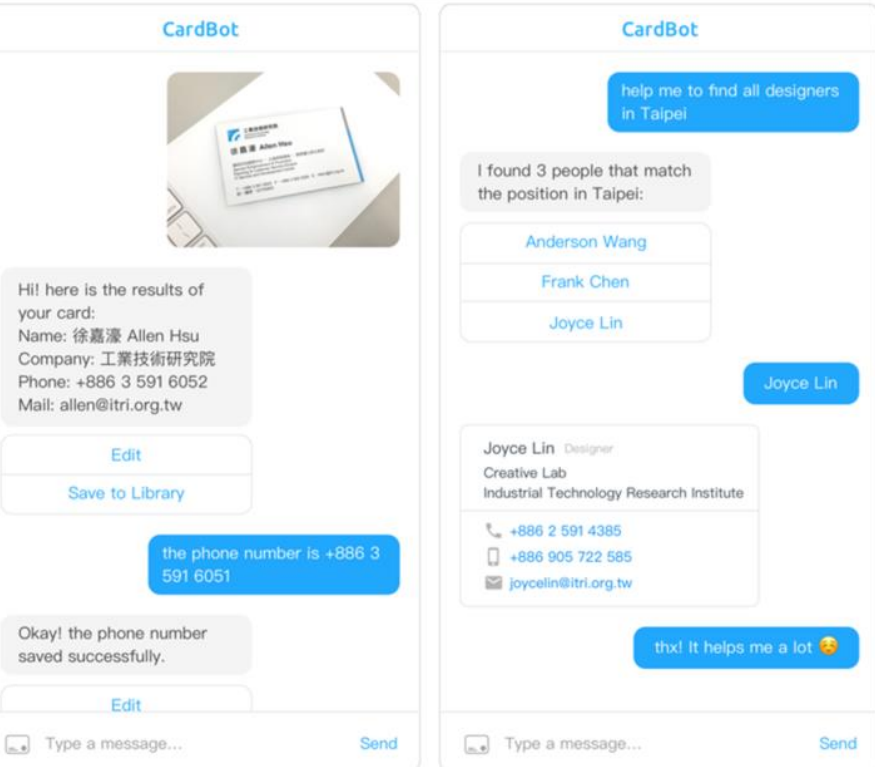

Figure 6: Cardbot

By using NLP named entity recognition technique, it can identify the key words such as, 'how much' and 'red chair'. After extracting the key words, those words will then be processed and will be searched thoroughly in the database as the chatbot's knowledge base will be primarily from the database. After understanding and processing the text, the chatbot can give responses. On the other hand, using NLP sentiment analysis technique can help the chatbot identify whether the interlocutor is feeling great or not about the products or services. This technique will be helpful especially when chit-chatting so the chatbot's attitude of replying can be more accurate, appropriate, and human-like.

Lastly, the training type of the chatbot will be better if it is pre-trained with datasets because it will be more prepared that way. If the chatbot relies on what was written on the website just like SuperAgent chatbot then the website has to be opened beforehand so that the chatbot could read what was written and it is not that efficient. However, OCR technique can be implemented for business chat bots if needed. The datasets will consist of business related datasets (about products or services) and daily conversation datasets so the chatbot could handle both questions related to the business side and daily conversations. These datasets then will be processed by NLP techniques mentioned in the second paragraph and resulted in contextual replies.

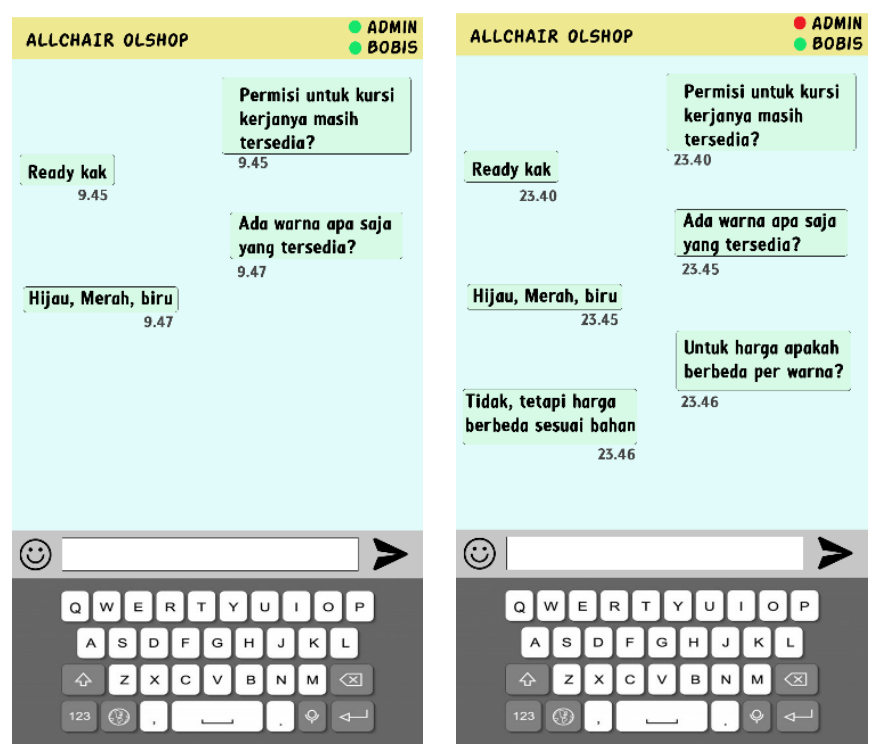

Figure 7: Prototype Chat Example

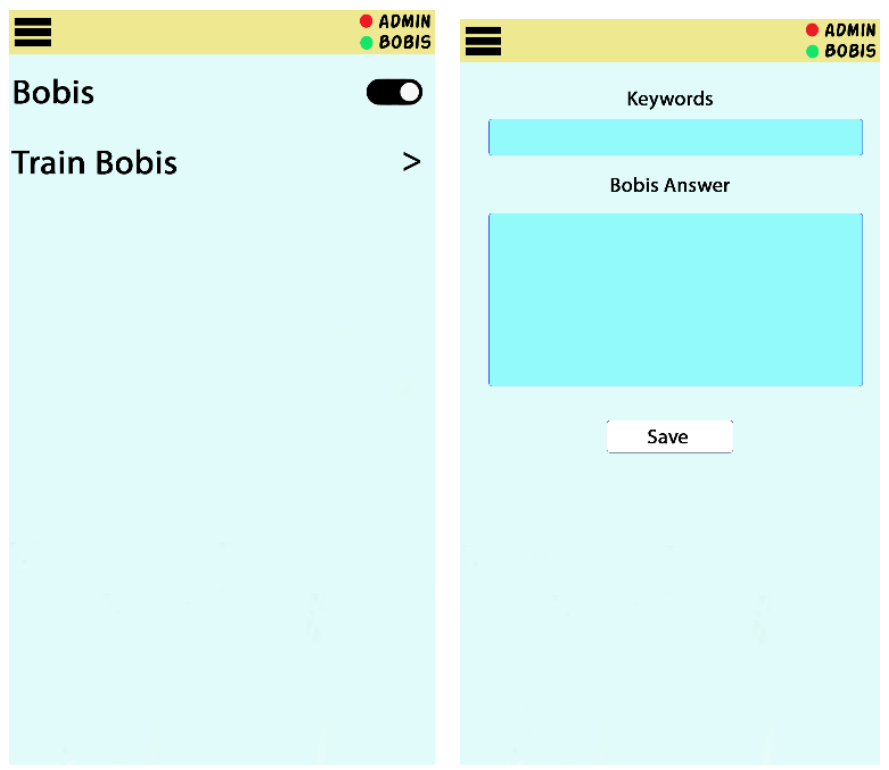

Figure 8: Prototype Setting and Training Menu Example

\subsection{Prototype Corresponding to The Result}

Based on the results our team got from our research, the chatbot should be able to be online 24 hours a day. The prototype that corresponds to this feature is depicted in Figure 7 where the chatting process could take place at any time and the chatbot would kindly reply. The other feature is that the chatbot should be able to work together with the seller just like an assistant would do, thus the chatbot can help replying to customers' chats when the seller is online or offline. For example, when there are a lot of chats coming through and the seller is online, but the seller only 
can reply one chat at a time. In that situation, chatbot could help replying to the other chats that the seller could not cover.

The seller also can train the chatbot outside the datasets they were trained before if needed. The feature is depicted in Figure 8 where the seller could give the keywords and what to answer towards that given keywords. This feature is just an additional feature so that the chatbot could study other keywords other than what it had been trained before. The home menu page depicted in Figure 9 is how the seller would see when it is full of customers' chats.

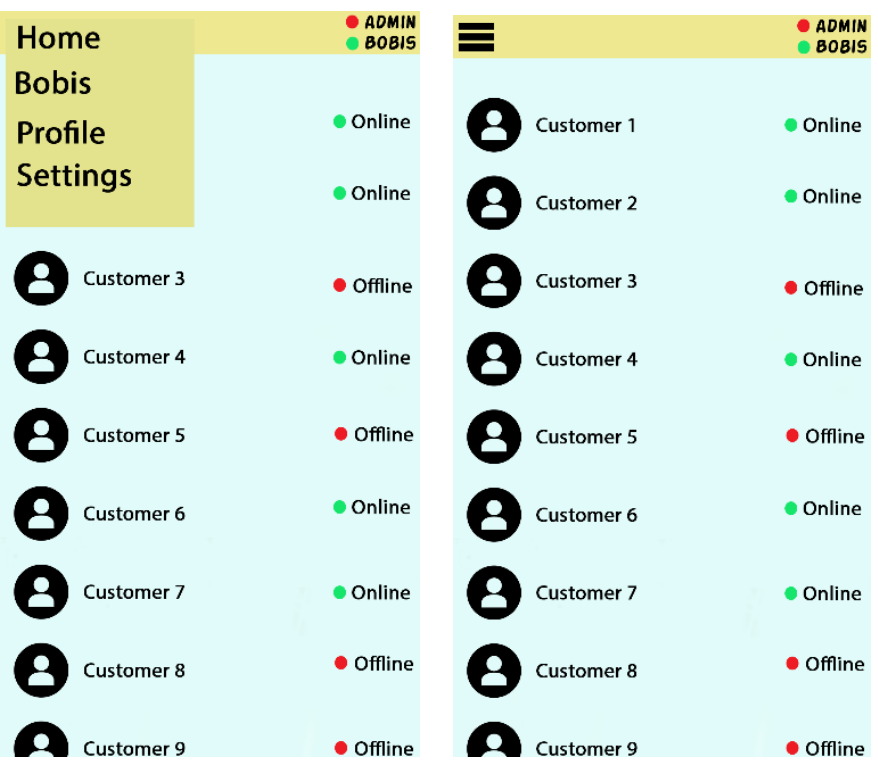

Figure 9: Prototype Home Menu Example

\section{Conclusion}

From the research that we did, we can conclude that there are some features that chatbots in a business environment need to have. First, the chatbot needs to be fast and accurate when answering questions and the information in the knowledge base need to be up to date. Then, the chatbot needs to have a text processing technique to help it process texts and thus we can use NLP named entity recognition technique and NLP sentiment analysis technique. The chatbot also needs to be ready 24 hours a day and should act like an assistant helping the seller rather than handling the online business alone. The datasets used should consist of daily conversation dataset and business-related dataset (about products or services). The datasets can be expanded if there are much more datasets needed in the business. The chatbot should be flexible when using, thus the self-training feature by the seller can be used as an additional feature inside the chatbot. However, the self-training feature by the seller can also be removed if the seller does not need it. Lastly, the chatbot interface should be simple as its main aim is to help sellers to enhance their online business. We also can conclude that there is a high chance of acceptability for the chatbot technology especially from younger people $[24,25]$.

For the future implementation of the chatbot, we recommend that the chatbot should become a part of the application or website the owner has. Since the chatbot should be able to work like an assistant in the business thus the chatbot should be placed in places that are easy to find. If the chatbot is implemented in ecommerce business, it would be convenient to have a chatting menu on the navigation bar and in each page of products respectively. If the chatbot is implemented in company websites, it would be convenient to have the chatting page relatively small to medium size and keep it on a fixed position on the page like a pop-up. Make sure to make it not too eye-catching nor too boring or else users may lose their attention to the chatting pop-up or even not knowing that the chatting pop-up is there. The selftraining feature of the chatbot should be implemented in another platform (not a part of the application or website) because we would not want any people controlling the data set without owners' consent.

\section{Conflict of Interest}

This paper is created due to a project in our research class and this is our group's research idea about chatbot in the business environment.

\section{Acknowledgment}

Special thanks to our lecturer Mrs. Azani that is willing to help us in writing this paper and our respondents that participated in giving their honest opinion for this research.

\section{References}

[1] R. Sutoyo, A. Chowanda, A. Kurniati, R. Wongso“Designing an emotionally realistic chatbot framework to enhance its believability with AIML and information states," Procedia Computer Science, 157, 621-628, 2019, doi:10.1016/j.procs.2019.08.226.

[2] G.D. Souza, "Chatbot for Organizational FAQs," (May), 5591-5594, 2019.

[3] S.A. Abdul-Kader, J.C. Woods, "Survey on Chatbot Design Techniques in Speech Conversation Systems," International Journal of Advanced Computer Science and Applications, 6(7), 72-80, 2015, doi:10.14569/ijacsa.2015.060712.

[4] N. Haristiani, "Artificial Intelligence (AI) Chatbot as Language Learning Medium: An inquiry,” Journal of Physics: Conference Series, 1387(1), 2019, doi:10.1088/1742-6596/1387/1/012020.

[5] Q.N. Nguyen, A. Sidorova, "Understanding user interactions with a chatbot: A self-determination theory approach," 2018.

[6] E. Bittner, O. Shoury, "Designing Automated Facilitation for Design Thinking: A Chatbot for Supporting Teams in the Empathy Map Method," Proceedings of the 52nd Hawaii International Conference on System Sciences, 227-236, 2019, doi:10.24251/hicss.2019.029.

[7] E. Yuniar, H. Purnomo, "Implementasi Chatbot 'Alitta' Asisten Virtual Dari Balittas Sebagai Pusat Informasi Di Balittas," Antivirus: Jurnal Ilmiah Teknik Informatika, 13(1), 24-35, 2019, doi:10.35457/antivirus.v13i1.714.

[8] M. Heo, K.J. Lee, "Chatbot as a New Business Communication Tool: The Case of Naver TalkTalk," Business Communication Research and Practice, 1(1), 41-45, 2018, doi:10.22682/bcrp.2018.1.1.41.

[9] L. Cui, S. Huang, F. Wei, C. Tan, C. Duan, M. Zhou, "Superagent: A customer service chatbot for E-commerce websites," ACL 2017 - 55th Annual Meeting of the Association for Computational Linguistics, Proceedings of System Demonstrations, 97-102, 2017, doi:10.18653/v1/P17-4017.

[10] M.C. Ko, Z.H. Lin, "Cardbot: A chatbot for business card management," International Conference on Intelligent User Interfaces, Proceedings IUI, 12, 2018, doi:10.1145/3180308.3180313.

[11] P. Smutny, P. Schreiberova, "Chatbots for learning: A review of educational chatbots for the Facebook Messenger," Computers and Education, 151(June 2019), 103862, 2020, doi:10.1016/j.compedu.2020.103862.

[12] N.S. Raj, B.R. Suteja, "Implementasi AIML pada Pandorabot untuk studi kasus Fakultas Teknologi Informasi," 1, 13-22, 2019.

[13] B. Mohit, Named entity recognition, Springer: 221--245, 2014, doi:10.1007/978-3-642-45358-8_7.

[14] T. Luo, S. Chen, G. Xu, J. Zhou, Sentiment Analysis, Springer: 53--68, 2013, doi:10.1007/978-1-4614-7202-5_4. 
[15] L. Abualigah, M.Q. Bashabsheh, H. Alabool, M. Shehab, "Text Summarization: A Brief Review," Studies in Computational Intelligence, 874(December 2019), 1-15, 2020, doi:10.1007/978-3-030-34614-0_1.

[16] M. Allahyari, S. Pouriyeh, M. Assefi, S. Safaei, E. D., J. B., K. Kochut, "Text Summarization Techniques: A Brief Survey," International Journal of Advanced Computer Science and Applications, 8(10), 2017, doi:10.14569/ijacsa.2017.081052.

[17] K. Vivekanandan, J.S. Araviandan, "Aspect-based Opinion Mining: A Survey," International Journal of Computer Applications, 106(3), 21-26, 2014.

[18] P. Kherwa, P. Bansal, "Topic Modeling: A Comprehensive Review," ICST Transactions on Scalable Information Systems, 0(0), 159623, 2018, doi:10.4108/eai.13-7-2018.159623.

[19] S.R. Joseph, H. Hloman, K. Letsholo, K. Sedimo, "Natural Language Processing: A Review," International Journal of Research in Engineering and Applied Sciences, 6(3), 1-8, 2016.

[20] A. Kawtrakul, "An Overview of a Role of Natural Language Processing in An Intelligent Information Retrieval System Conventional IR V . S . Intelligent IR."

[21] B. Setiaji, F.W. Wibowo, "Chatbot Using a Knowledge in Database: Humanto-Machine Conversation Modeling," Proceedings - International Conference on Intelligent Systems, Modelling and Simulation, ISMS, 0, 7277, 2016, doi:10.1109/ISMS.2016.53.

[22] M. Nuruzzaman, O.K. Hussain, "IntelliBot: A Dialogue-based chatbot for the insurance industry," Knowledge-Based Systems, 196(xxxx), 105810, 2020, doi:10.1016/j.knosys.2020.105810.

[23] R. Sankar, "Empowering Chatbots With Business Intelligence By Big Data Integration," International Journal of Advanced Research in Computer Science, 9(1), 627-631, 2018, doi:10.26483/ijarcs.v9i1.5398.

[24] M. Kottorp, F. Jäderberg, "Chatbot as a potential tool for businesses A study on chatbots made in collaboration with Bisnode," 2017.

[25] M. Van Eeuwen, "Mobile conversational commerce: messenger chatbots as the next interface between businesses and consumers," University of Twente, 15, 2017. 